\title{
On the Parameterization of Surface Roughness at Regional Scales
}

\author{
Elie Bou-Zeid and Marc B. Parlange \\ School of Architecture, Civil, and Environmental Engineering, Ecole Polytechnique Fédérale de Lausanne, Lausanne, Switzerland, \\ and Department of Geography and Environmental Engineering, and Center for Environmental and Applied Fluid Mechanics, The \\ Johns Hopkins University, Baltimore, Maryland \\ Charles Meneveau \\ Department of Mechanical Engineering, and Center for Environmental and Applied Fluid Mechanics, The Johns Hopkins University, \\ Baltimore, Maryland
}

(Manuscript received 10 November 2005, in final form 19 April 2006)

\begin{abstract}
A parameterization for surface roughness and blending height at regional scales, under neutral atmospheric stability, is studied and tested. The analysis is based on a suite of large-eddy simulations (LES) over surfaces with varying roughness height and multiple variability scales. The LES are based on the scaledependent Lagrangian dynamic subgrid-scale model, and the surface roughnesses at the ground are imposed using the rough-wall logarithmic law. Several patterns of roughness distribution are considered, including random tiling of patches with a wide distribution of length scales. An integral length scale, based on the one-dimensional structure function of the spatially variable roughness height, is used to define the characteristic surface variability scale, which is a critical input in many regional parameterization schemes. Properties of the simulated flow are discussed with special emphasis on the turbulence properties over patches of unequal roughness. The simulations are then used to assess a generalized form of the parameterization for the blending height and the equivalent surface roughness at regional scales that has been developed earlier for regular patterns of surface roughness (regular stripes). The results are also compared with other parameterizations proposed in the literature. Good agreement is found between the simulations and the regional-scale parameterization for the surface roughness and the blending height when this parameterization is combined with the characteristic surface variability scale proposed in this paper.
\end{abstract}

\section{Introduction}

Due to the complex physics that govern turbulent atmospheric boundary layer (ABL) flows, most of the approaches developed to describe this layer are empirically formulated using similarity theory and field data (Stull 1988). The surface layer, which covers the bottom $10 \%-20 \%$ of the $\mathrm{ABL}$, is the region where most similarity theories have been developed. The MoninObukhov (MO) similarity theory (Monin and Obukhov

Corresponding author address: Elie Bou-Zeid, School of Architecture, Civil, and Environmental Engineering, Ecole Polytechnique Fédérale de Lausanne, EFLUM, GR A0 412, station 2, CH-1015 Lausanne, Switzerland.

E-mail: eliebz@jhu.edu

DOI: $10.1175 / J A S 3826.1$

(C) 2007 American Meteorological Society
1954) is very well suited for that layer when the wind shear is nonnegligible (negligible wind shears corresponding to free convection conditions are a rather rare occurrence in the surface layer). In fact, MO similarity remains the primary approach for the computation of regional-scale surface fluxes using measurements in the atmospheric surface layer and for the parameterization of the lower ABL in large-scale weather and climate models (Taylor 1987; Mason 1988; Claussen 1991; Parlange et al. 1995; Bou-Zeid et al. 2004).

The MO similarity was developed for homogeneous surfaces, but it has been commonly used for landatmosphere interaction over variable surfaces at regional scales. The applicability of this similarity theory over variable surfaces can be attributed to the strong mixing effects of atmospheric turbulence and to the 
small extent of vertical length scales vis-à-vis horizontal length scales (Brutsaert 1998). Field experiments found the similarity theory to be accurate in obtaining the regional-scale evaporation, momentum flux, and sensible heat flux over variable surfaces that appear statistically homogeneous at the regional scale (Kustas and Brutsaert 1986; Parlange and Brutsaert 1989, 1993; Sugita and Brutsaert 1990, 1992).

For momentum flux computations at regional scales, an effective regional surface roughness $\left(z_{o, e}\right)$ can be obtained from the log-law based on wind profiles measured under a near-neutral atmospheric stability:

$$
\langle u\rangle=\frac{u_{*}}{\kappa} \ln \left(\frac{z}{z_{o, e}}\right),
$$

where $\kappa=0.4$ is the von Kármán constant, $\langle u\rangle$ is the time-averaged streamwise velocity, $u_{*}$ is the friction velocity, $z$ is the height above ground, and $z_{o, e}$ is the effective surface roughness. The issue of specifying the effective surface roughness is important for mesoscale and global circulation modeling where significant variations in surface roughness occur at scales smaller than the grid size (Avissar and Pielke 1989; Houghton et al. 1997; Koster and Suarez 1992; Dai et al. 2003). Often, average wind profiles, at regional scale and under neutral stability, are not available for determining $z_{o, e}$, and it would be useful if a procedure was available to scale local surface roughnesses up to the regional scale.

Several approaches have been attempted to compute effective roughness for natural landscapes based on simple surface information. Methods relying simply on the spatial average of surface roughness of landscape patches (Taylor 1987) do not necessarily capture the effects of abrupt changes in surface conditions. Other approaches use higher order statistics such as frequency distributions (Avissar and Pielke 1989; Avissar 1991, 1992); these approaches do not account for variability scales and interactions between patches. It will be shown in this paper that simulations with the same univariate probability density function (PDF) of surface roughness can yield different land-atmosphere interaction dynamics (as depicted by the domain averaged velocities for example), due to changes in the variability scale and the resulting variations in patch interactions. Other approaches that do include variability scale in the parameterizations (Mason 1988; Claussen 1990, 1991; Goode and Belcher 1999; Bou-Zeid et al. 2004) are expected to be more successful.

To compute effective surface roughness, Bou-Zeid et al. (2004, hereafter BMP04) recently derived two equations relating the effective surface roughness $z_{o, e}$, and the blending height $h_{b}$. The first equation tracked the growth of internal boundary layers emanating from the boundaries of the patches; the approach used observations (from LES simulations) that blending occurs only when these internal boundary layers have evolved over downstream distances about twice the characteristic length scale. The second equation expressed the total surface force over a variable area as the discrete sum of surface forces over the individual homogeneous patches composing that area, each of the $N$ patches having roughness $z_{o, i}$ and area fraction $f_{i}(i=1,2$, $3, \ldots, N)$. The two equations were then combined to yield one equation that can be solved iteratively for the blending height $h_{b}$ :

$$
\left(\frac{h_{b}}{1.7 \kappa L_{p}+h_{b}}\right)^{2}=\sum_{i=1}^{N}\left[f_{i} /\left(\ln \frac{h_{b}}{z_{\mathrm{o}, i}}\right)^{2}\right] .
$$

Subsequently, the value of the effective surface roughness, $z_{o, e}$, is computed from:

$$
z_{o, e}=h_{b} \exp \left(-\frac{1.7 \kappa L_{p}}{h_{b}}-1\right),
$$

where $L_{p}$ is a characteristic variability scale. As a first assessment of the parameterization, BMP04 performed 20 high-resolution $\left(120^{3}\right.$ nodes) LES simulations of ABL flow over idealized cross-stream stripes of varying surface roughness. All stripes had the same streamwise length such that only one surface variability scale existed in each simulation: the streamwise length of the patches. Agreement between the LES and the parameterization was good and the parameterization was shown to give better estimates of $z_{o, e}$ than the logaverage (Taylor 1987) or the parameterization of Mason (1988). The parameterization is very easy to implement for very complex distributions and when the surface properties are remotely sensed from airplanes or satellites. The effect of changes in roughness is accounted for in that parameterization only in an average sense through the variability scale, $L_{p}$, that characterizes the number of changes in roughness.

Nevertheless, several basic questions remained unanswered in that work and have rarely, if ever, been addressed in the literature dealing with regional-scale parameterizations:

1) Will the parameterization work for complex patches and for surfaces exhibiting multiple variability scales? Most previous parameterizations of regional-scale surface roughness were formulated and tested for very simple configurations featuring a few 
patches and a single variability scale (Taylor 1987; Mason 1988; Claussen 1990; Goode and Belcher 1999; BMP04). Since abrupt changes in roughness are critical for land-atmosphere momentum exchange (Albertson and Parlange 1999b), generalization and validation of regional scale parameterizations are needed for realistically complex surfaces with multiple variability scales.

2) If regional parameterizations are adequate for complex surfaces, what variability length scale $\left[L_{p}\right.$ in Eqs. (2) and (3)] should be used to represent the various variability scales actually featured in real surfaces? BMP04, as well as other regional parameterizations (Mason 1988; Claussen 1990; Goode and Belcher 1999), require an average patch length but none of the studies address how such a scale can be derived for realistic surfaces (e.g., from satellite data) and none test their proposed parameterization with such an aggregate variability scale. Characteristic variability scales are also needed in numerous other applications dealing with variable surfaces (see Brutsaert 1998).

The main aim of this paper is to address these two remaining questions. To that end, a suite of ABL simulations is performed using the large-eddy simulation technique with a new-generation model for subgridscale (SGS) turbulence that is appropriate for variable surfaces. The next section of the paper presents the large-eddy simulation model used in this study. Then, the suite of simulations performed is presented. Basic properties of the simulated flow, such as turbulence properties over patches of unequal roughness, are discussed. The parameterization for the effective surface roughness $\left(z_{o, e}\right)$ and blending height $\left(h_{b}\right)$ proposed in BMP04 is then generalized by the introduction of a characteristic spatial variability scale for applications of BMP04 (and other parameterizations) to realistically complex surfaces. Finally, the generalized parameterization for $z_{o, e}$ and $h_{b}$ is successfully tested for the complex surfaces simulated in this study.

\section{Large-eddy simulation code}

Large-eddy simulation has become an important tool for the study of high Reynolds number environmental (Deardorff 1974; Moeng 1984; Shaw and Schumann 1992; Albertson and Parlange 1999a, 1999b; Wood 2000; BMP04) and engineering (Lesieur and Metais 1996; Piomelli 1999; Sagaut 2006) turbulent flows. The basic premise in LES is that the largest eddies contain most of the energy and are responsible for most of the transport of momentum and scalars.
Hence, the LES technique consists of resolving only these large scales of motion and filtering out eddies smaller than the grid-filter size, reducing the numerical cost of the technique considerably and enabling the simulation of high Reynolds number flows. The effect of the unresolved scales cannot be discarded and appears as an additional term in the filtered NavierStokes equations. This term involves the divergence of an additional unknown, the subgrid-scale stress tensor, and hence the turbulence closure problem emerges. To close the system of equations, a model for the subgridscale stress is required. The results of large-eddy simulations are quite sensitive to this model, especially in the vicinity of solid boundaries where the subgrid-scale fluxes are important and their physics are harder to model [see a discussion in Meneveau and Katz (2000), and an illustration in Bou-Zeid et al. (2005)]. The most widely used model for the subgrid-scale stress remains the Smagorinsky (1963) model, which relates the subgrid-scale stress to the resolved strain rate tensor via an eddy viscosity. Lilly (1967) computed the eddy viscosity for homogeneous isotropic turbulence. Germano et al. (1991) proposed a method to dynamically compute the eddy viscosity from information about the smallest resolved scales, thus allowing the modeling of nonhomogeneous and anisotropic turbulence. The need for some kind of averaging became obvious with the dynamic model of Germano to preserve numerical stability and to recover the statistical basis of the eddy viscosity formulation (Meneveau and Katz 2000). Meneveau et al. (1996) proposed an approach to carry this averaging over fluid pathlines and, thus, preserve local variability and allow the simulation of complex flows with heterogeneous boundaries. The application of the Lagrangian dynamic model in LES codes using wall models (as opposed to LES that resolve the viscous sublayer) remained problematic near solid boundaries where the smallest resolved scales loose their universality due to the effect of the boundary. To correct this problem, the model needs to account for the scale-dependence of these smallest resolved scales in the vicinity of solid walls (Porte-Agel et al. 2000; Kleissl et al. 2003, 2004). Bou-Zeid et al. (2005) proposed an efficient formulation to measure this scale-dependence dynamically; particularly, this formulation worked well with the Lagrangian averaging approach. In this work, we use the dynamic, scale-dependent, Lagrangian model detailed in Bou-Zeid et al. (2005). The model reproduces the log-law near the ground and the streamwise velocity spectra follow the experimental and theoretical results, suggesting that the model is successfully capturing the coupling between the atmosphere and the earth's sur- 
TABLE 1. Simulation characteristics.

ABL height, $H$

Horizontal domain size $L_{x} \times L_{y}$

Vertical mesh spacing, $d z$

Horizontal mesh spacing, $d x$ and $d y$

Number of grid points

Initial conditions

Warm-up period

Simulation time step

Number of simulation time steps

Output sampling frequency

Friction velocity
$1000 \mathrm{~m}$

$6280 \mathrm{~m} \times 6280 \mathrm{~m}$

$8.4 \mathrm{~m}$; some variables are stored at $(n-1 / 2) d z$

$\sim 52 \mathrm{~m}$

$120^{3} \sim 1.7$ million points

Modified logarithmic profile with a randomly imposed turbulent kinetic energy (TKE)

Warm-up simulations are run until the vertical stress profile is linear and the total kinetic energy in the domain is stable

About $0.25 \mathrm{~s}$ if a friction velocity $u_{*}$ of $0.5 \mathrm{~m} \mathrm{~s}^{-1}$ is assumed

100000 (for a total physical simulation time of about $8 \mathrm{~h}$ )

Every 10 time steps

Defined through the imposed streamwise pressure gradient $d P / d x$ as $u_{*}=\sqrt{-\frac{d P}{d x} \frac{H}{\rho L_{x}}}$,
where $\rho$ is the fluid density face. The model also performed well in tests over variable surfaces reproducing properly the velocity and stress adjustments observed after a change in surface roughness by Bradley (1968).

The numerical code uses a pseudospectral approach in the horizontal directions and consequently the horizontal boundary conditions are periodic; that is, the flow that exits at the downstream boundary of the domain is fed back at the upstream boundary. A secondorder accurate centered differences scheme is applied in the vertical direction. The fully explicit second-order accurate Adams-Bashforth scheme is used for time advancement. Full dealiasing of the convective terms is performed using the $3 / 2$ rule (Orszag 1970, 1971). The pressure is computed from a Poisson equation obtained by setting the divergence of the momentum equation to zero. This is equivalent to (and hence substitutes for) solving the continuity equation.

Stress free and no-penetration conditions are imposed at the top of the domain by setting $\partial_{3} \tilde{u}_{1,2}=\tilde{u}_{3}=$ 0 , where 1,2 , and 3 (or $x, y, z$ in other parts of the paper) refer to the streamwise, cross-stream, and vertical directions, respectively. This means that the flow being modeled is actually a pressure-driven channel flow; nevertheless, since this paper is concerned with dynamics occurring in the bottom part of the ABL, Coriolis effects are negligible and the simulations are good representations of ABL flow.

At the bottom of the domain, the vertical velocity is set to 0 and surface stresses are imposed through a local law-of-the-wall formulation (Deardorff 1970); however, velocities filtered at twice the grid scale are used to compute the surface stress; this is needed to ensure that the average stress over the wall is close to the stress predicted by the classic log-law (see Bou-Zeid et al. 2005 for details). A sharp spectral cutoff filter is used for the wall model and SGS model filtering operations.

\section{Simulations}

LES provides only the resolved part of the velocities and stresses; this resolved part is denoted by a tilde. A typical $1 \mathrm{~km}$ deep ABL is simulated; the horizontal size of the domain is $6.28 \mathrm{~km}$ by $6.28 \mathrm{~km}$. Table 1 lists the various details of the simulations such as resolution, forcing, initialization, and output sampling frequency.

Two sets of simulations were performed. The first set consisted of eight simulations of flow over two unequal cross-stream stripes with different surface roughness $\left(z_{o, 1}=0.01 \mathrm{~m}\right.$ and $\left.z_{o, 2}=0.1 \mathrm{~m}\right)$; an infinite series of stripes is effectively simulated owing to the periodic horizontal boundary conditions used in the code. Figure 1 shows the simulation domain corresponding to the simulation where the rougher patch covered $75 \%$ of the domain and the smoother patch the remaining $25 \%$ (hence the name of that simulation S25R75 and the acronym SR used to refer to that series of simulations). The length of the high-roughness patch was systematically increased, and hence the length of the lowroughness patch was correspondingly decreased, to simulate surfaces with a range of effective surface

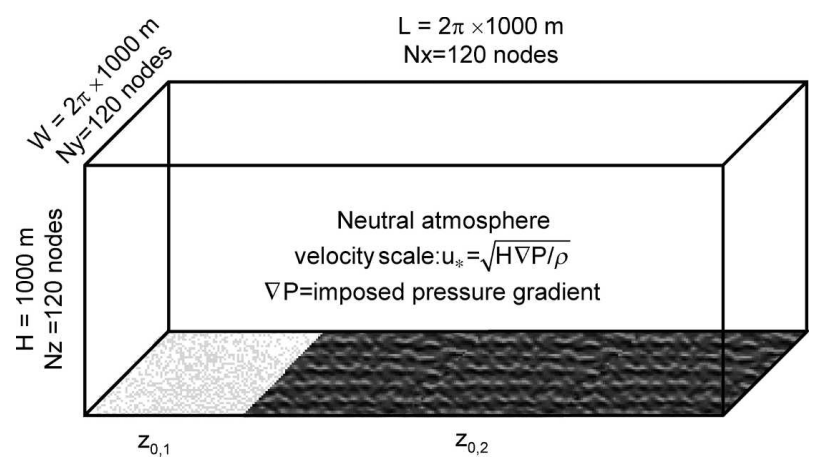

FIG. 1. Computational domain with the surface corresponding to simulation S25R75 (not to scale). 
TABLE 2. Simulation scenarios for surfaces with regular stripes of unequal lengths.

\begin{tabular}{lcrrccc}
\hline \hline Simulation & $\begin{array}{c}\text { No. of } \\
\text { patches }\end{array}$ & $\begin{array}{c}L_{p 1} \\
(\mathrm{~m})\end{array}$ & $\begin{array}{c}L_{p 2} \\
(\mathrm{~m})\end{array}$ & $\begin{array}{c}z_{o, 1}, z_{o, 2} \\
(\mathrm{~m})\end{array}$ & $\begin{array}{c}z_{o, \mathrm{e}} \\
(\mathrm{m})\end{array}$ & $\begin{array}{c}h_{b} \\
(\mathrm{~m})\end{array}$ \\
\hline S07R93 & 2 & 440 & 5840 & $0.01,0.1$ & 0.090 & 225 \\
S12R88 & 2 & 750 & 5525 & $0.01,0.1$ & 0.080 & 230 \\
S25R75 & 2 & 1570 & 4710 & $0.01,0.1$ & 0.063 & 240 \\
S33R67 & 2 & 2070 & 4210 & $0.01,0.1$ & 0.058 & 240 \\
S67R33 & 2 & 4210 & 2070 & $0.01,0.1$ & 0.028 & 260 \\
S75R25 & 2 & 4710 & 1570 & $0.01,0.1$ & 0.023 & 265 \\
S88R12 & 2 & 5525 & 750 & $0.01,0.1$ & 0.017 & 305 \\
S93R07 & 2 & 5840 & 440 & $0.01,0.1$ & 0.014 & 310 \\
\hline
\end{tabular}

roughness as detailed in Table 2. Table 2 also lists the blending height determined using profiles of velocity quartiles and the effective surface roughness computed from average LES velocity profiles. More details about the methods used to compute the blending height and effective surface roughness from LES data can be found in BMP04. For these simulations, the variability scale, or patch length, was approximated as the average length of the patches (half the length of the domain $=$ $3.14 \mathrm{~km})$.

In the second set (referred to as the CS series, for complex surfaces), random configurations of patches were simulated. The configurations mimicked more closely real surfaces. However, to focus attention on the effect of the spatial variability scale, surfaces with different variability scales, but with the same statistical distribution of the roughness, were created. An initial surface was created with relatively large patches, the initial patches were placed manually in a fairly random arrangement, to create the relatively realistic-looking spatial pattern shown in Fig. 2. Then, the surface roughness (in centimeters) of these patches was assigned using a univariate lognormal random number generator (from MATLAB ${ }^{\mathrm{TM}}$ ) with $\mu=\log (20)$ and $\sigma=\log (10)$, the resulting roughness heights, $z_{o}$, were clipped at 100 $\mathrm{cm}$ so all values of $z_{o}$ ranged between 0 and $100 \mathrm{~cm}$ (Fig. 2). Note that, since we are using a lognormal random number generator, $\mu$ and $\sigma$ are not the mean and standard deviation of the generated populations.

Subsequently, the surface in Fig. 2 was subdivided into a matrix of square regions (see Fig. 3); the squares were then ordered (numbered) consecutively from top left to bottom right; the order of the squares was then randomly shuffled (using the MATLAB "randperm" function), and the new surface was created by pasting (from top left to bottom right) the squares following the new shuffled order. The smaller the square divisions are, the smaller the resulting characteristic variability scale will be. Hence, six additional surfaces were created from the original surface and all of the surfaces

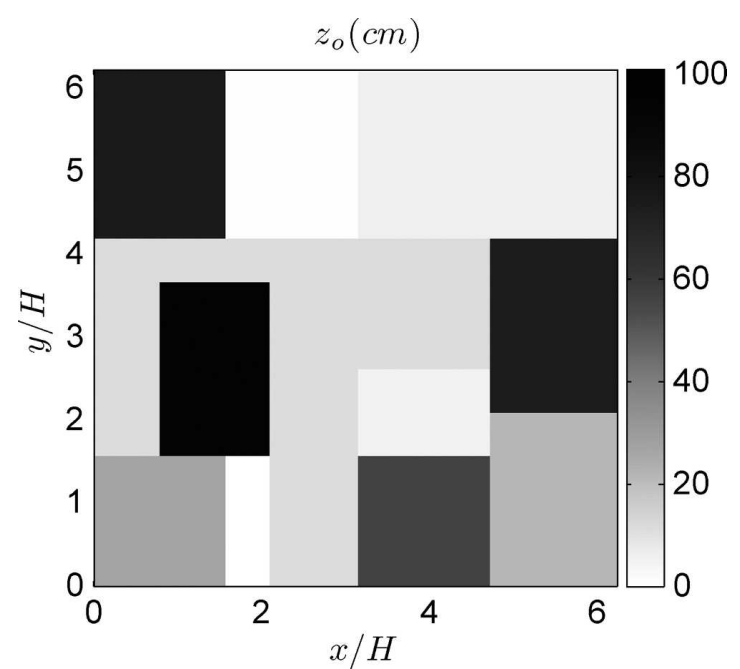

FIG. 2. Complex patch configuration with random surface roughness (surface CS1), axis in this figure, and in all figures of the paper, are normalized by the boundary layer height $H=1000 \mathrm{~m}$.

were then simulated. The seven surfaces have the same univariate PDF in $z_{o}$ but with different spatial distribution and with a decreasing trend of the variability scale $L_{p}$

The main results measured from the LES were the effective surface roughness and blending height. These were determined from the simulated fields following the same procedures as in BMP04. The blending height is determined from the vertical profiles of the upper and lower velocity quartiles as the height where the two profiles merge and no longer oscillate significantly, indicating a fairly homogeneous flow. The effective roughness is computed, using a least-square error fit of a log-law of the form of Eq. (1) with $\kappa=0.4$, from the mean streamwise velocity profiles in the lowest $60 \mathrm{~m}$, averaged over time as well as in the horizontal plane $(x, y)$. Results are presented in Tables 2 and 3. To compare these LES results with predictions from the BMP04 parameterization, a characteristic length scale for horizontal variability is still required. This is discussed in the next section.

\section{Characteristic variability scale}

A possible approach to measure the variability scale for some generic surface characteristic $\gamma$ (such as skin temperature or surface roughness) is to compute the two-point covariance function

$$
C_{\gamma}\left(r_{t}\right)=\left\langle\left(\gamma\left(r+r_{t}\right)-\langle\gamma\rangle\right)(\gamma(r)-\langle\gamma\rangle)\right\rangle
$$

or the structure function

$$
D_{\gamma}\left(r_{t}\right)=\left\langle\left(\gamma\left(r+r_{t}\right)-\gamma(r)\right)^{2}\right\rangle
$$




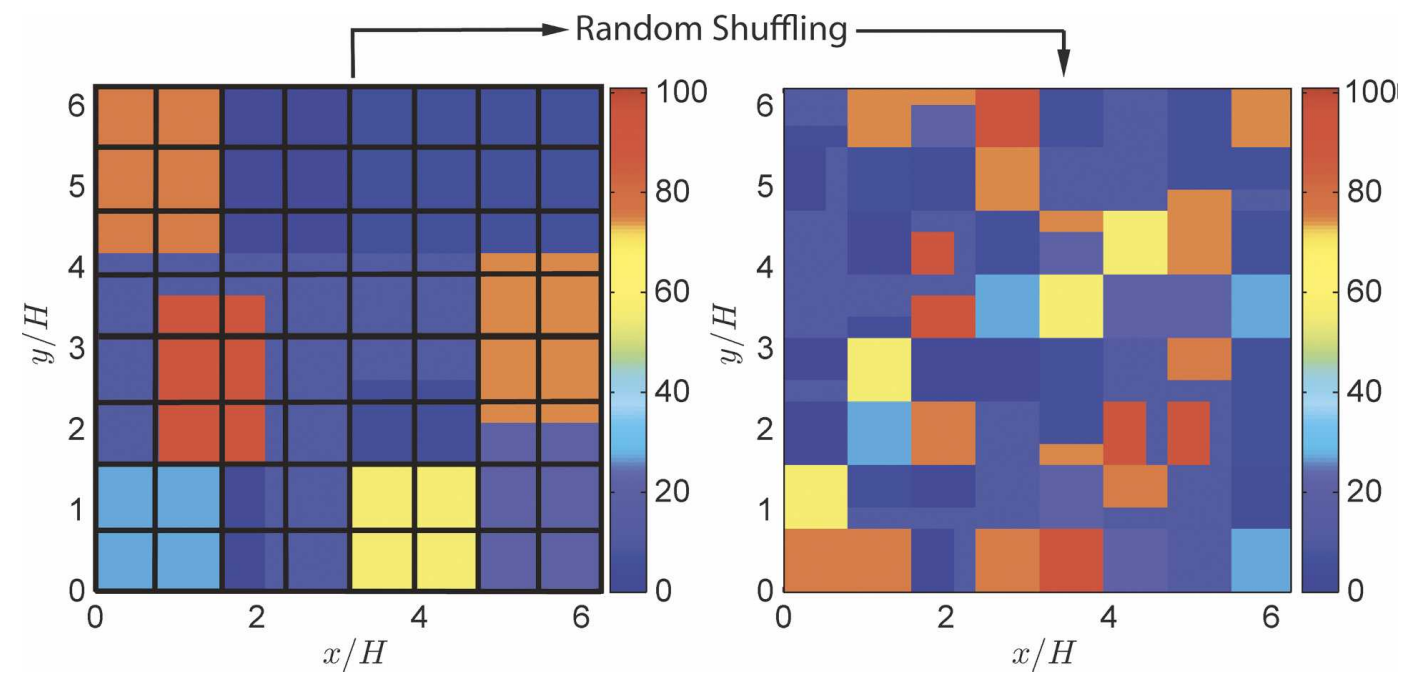

FIG. 3. Reconfiguration of the complex patch surface yielding a smaller variability scale; figure on the right corresponds to simulation CS4.

for increasing translation vectors $\mathbf{r}_{t}$ (Brutsaert 1998; Lyons and Halldin 2004). Note that the outer angle brackets denote averaging over the whole surface being considered. As the magnitude of $\mathbf{r}_{t}$ increases, the surface properties at locations $\mathbf{r}$ and $\mathbf{r}+\mathbf{r}_{t}$ become more independent. Therefore, the correlation will decrease initially and then reach a constant minimal value, while the structure function will increase and then reach a plateau. A characteristic length scale could then be defined as the length of the translation vector $\mathbf{r}_{t}$ at which the correlation reaches its minimum value or the structure function reaches the plateau. The two values of the variability scale should be quite similar except that the one based on the structure function will be more reliable for surfaces that are not statistically homogeneous; this occurs for example when the roughness length has some drift in a given direction (see Brutsaert 1998). Figure $4 \mathrm{~d}$ depicts three examples of one-dimensional structure functions (normalized by their maxima) based on surface roughness as a function of translation distance $r_{t}$ for the surfaces of simulations CS2 (Fig. 4a), CS3 (Fig. 4b), and CS5 (Fig. 4c). Here $r_{t}$ is taken in the direction of the mean flow.

Note that computing the variability scale based on the maximum of the structure function basically yields the largest variability scale of the surface. The structure function will keep increasing until $\left|\mathbf{r}_{t}\right|$ exceeds the length of the largest patch. For the surface in Fig. 4a, for example, this method yields a variability scale of about $2.2 \mathrm{~km}$, which clearly exceeds the size of most of the patches in the surface. Therefore, this method might not give a scale that is truly representative for surfaces that exhibit several variability scales, especially if these surfaces yield a structure function with several plateaus and peaks. In particular, for parameterizations of landatmosphere momentum exchanges, we are also interested in some of the smaller variability scales since each change in roughness will lead to increased momentum transfer at the earth surface and hence increase the effective roughness $z_{o, e}$ (Albertson and Parlange 1999b; BMP04). Another problem with the definitions of the characteristic length scale based on the covariance and structure functions is that, for heterogeneous surfaces that are not statistically homogeneous, the constant minimum or maximum plateaus do not exist. This approach only works if the covariance and variograms are spatially homogeneous, that is, if the surface property is homogeneous to second order. In addition, visual inspection would be needed to determine the peak and plateau and deduce the variability scale, which makes

TABLE 3. $L_{p}, h_{b}$, and $z_{o, e}$ for the different surfaces simulated.

\begin{tabular}{lccccccc}
\hline \hline & CS1 & CS2 & CS3 & CS4 & CS5 & CS6 & CS7 \\
\hline$L_{p}(\mathrm{~m})$ & 1542 & 1292 & 936 & 1062 & 630 & 442 & 11 \\
$z_{o, e}$ & 0.219 & 0.214 & 0.216 & 0.2178 & 0.2359 & 0.2577 & 0.3088 \\
$h_{b}$ & 200 & 150 & 140 & 115 & 95 & 60 & 35 \\
\hline
\end{tabular}


(a) $z_{o}$ for simulation $C S 2(\mathrm{~cm})$

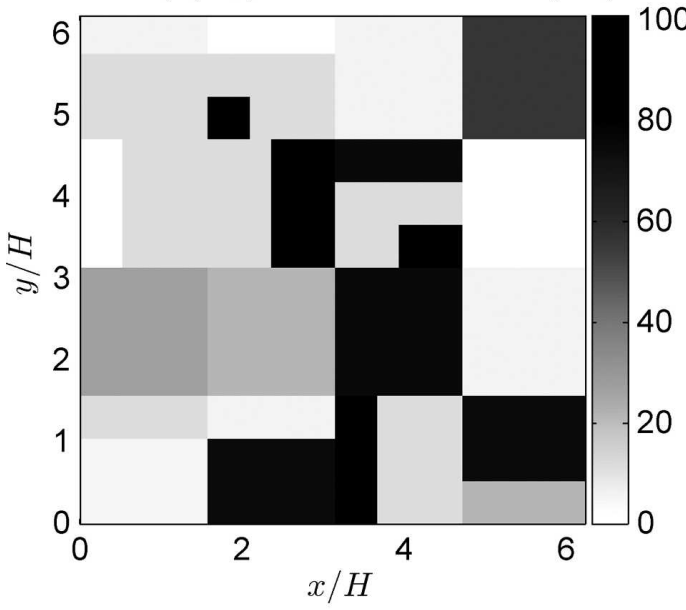

(c) $z_{o}$ for simulation $C S 6(\mathrm{~cm})$

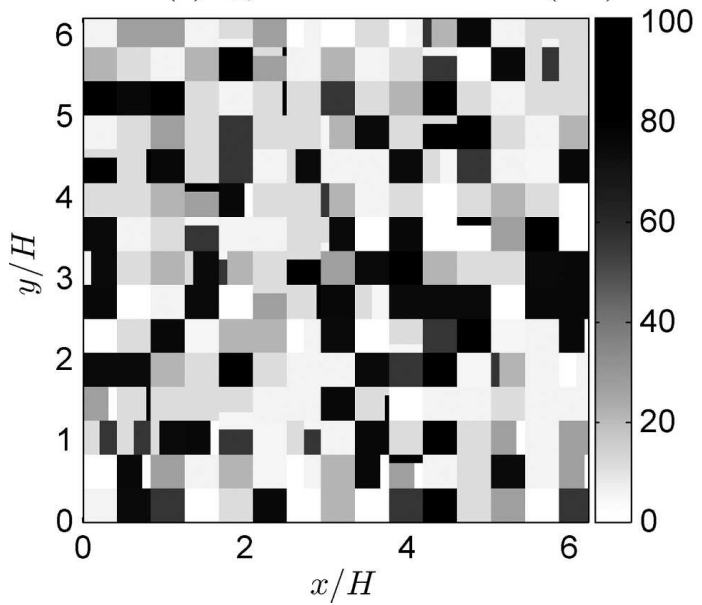

(b) $z_{o}$ for simulation $C S 3(\mathrm{~cm})$

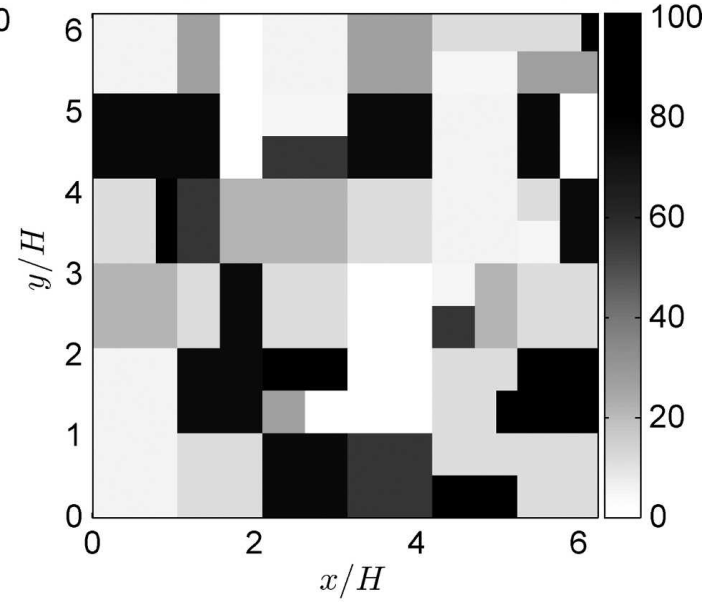

$(d)$

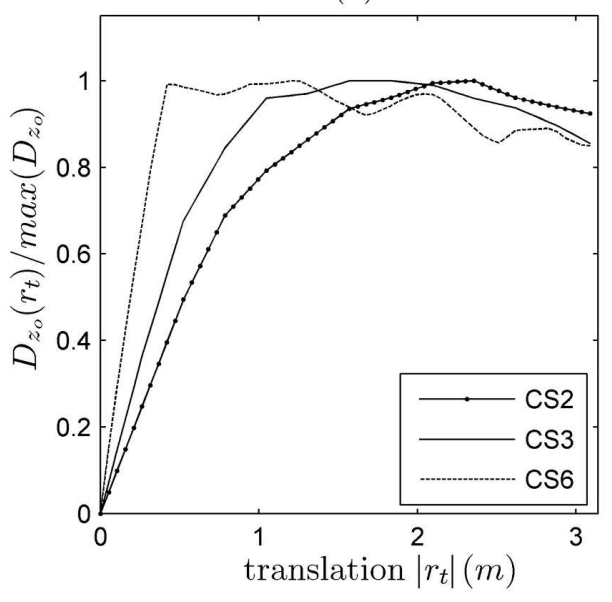

FIG. 4. Surface roughness for simulation (a) CS2, (b) CS3, and (c) CS5, and (d) the normalized structure functions based on surface roughness as a function of translation $\left|r_{t}\right|$.

this approach problematic to automate and hence tedious to use for each mesoscale or global model grid cell.

Another definition, also based on the structure function, can de developed by considering the integral length scale in a way similar to the integral scale of turbulence. It is appropriate to consider a translation vector $\mathbf{r}_{t}$ along the streamwise direction, with a magnitude $\left|\mathbf{r}_{t}\right|=r_{t}$. The characteristic variability scale, $L_{p}$, can then be defined as

$$
L_{p}=\int_{0}^{L_{D}}\left[1-\frac{D_{\gamma}\left(r_{t}\right)}{\max \left(D_{\gamma}\right)}\right] d r_{t},
$$

where $L_{D}$ is the streamwise length of the domain being considered. Note that this formulation will only be able to capture variability scales smaller than the integration limit, $L_{D}$. For simulation CS2, depicted in Fig. 4a, the characteristic scale given by Eq. (6) is about $1.1 \mathrm{~km}$, which is closer to the average size of the patches of that surface than the scale of $2.2 \mathrm{~km}$ computed based on the maximum of the structure function.

\section{Basic results}

Table 3 lists the variability scales, using the integral length scale definition of Eq. (6), for the different simulations. Also shown are the effective surface roughness lengths and blending heights, determined from LES profiles, for all seven simulations of flow over complex patches.

To illustrate the effect of the spatial variability scale on ABL dynamics, several basic properties of the simulated flow over complex patches are discussed. After a change in surface roughness, the flow starts to adjust to the new boundary conditions and this adjustment 


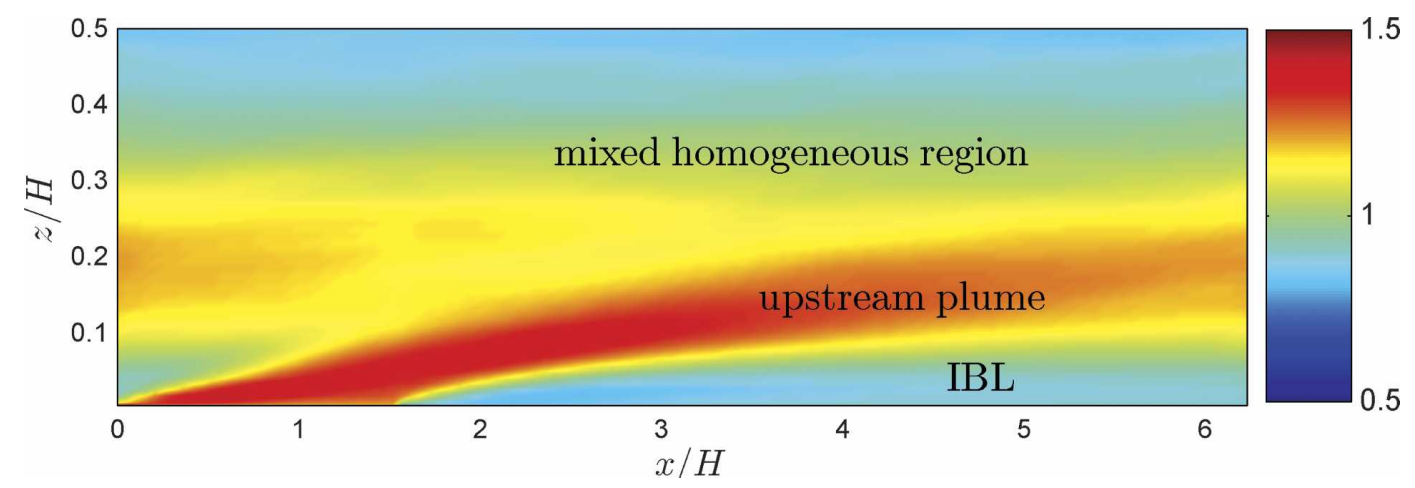

FIG. 5. Plot of $\left[\partial\langle\tilde{u}\rangle_{y, t} / \partial z-\partial\langle\tilde{u}\rangle_{x, y, t} / \partial z\right] H / u_{*}$ depicting the different layers in the ABL for simulation S25R75.

gradually propagates upward, defining an internal boundary layer (IBL). The depth of the IBL at a certain distance $x$ after the change in roughness is about $x / 10$ (Brutsaert 1998). Above the IBL, the effect of the underlying patch is insignificant; however, the flow is controlled by the properties of the upstream patches and it is not necessarily homogeneous. The "signatures" of the different patches are completely blended out farther up, at the so-called blending height $\left(h_{b}\right)$ above which the flow is statistically homogeneous. Note however that this blending height is not easily detectable. Blending occurs progressively and, as such, the blending height can vary depending on the criteria used to define it. For example, vertical stress profiles suggest a higher blending height than vertical velocity profiles (BMP04). This blending process can be visualized by plotting the deviation of the local mean velocity gradient in the vertical direction from its value averaged over all patches; that is, by plotting $\partial\langle\tilde{u}\rangle_{y, t} / \partial z-\partial\langle\tilde{u}\rangle_{x, y, t} /$ $\partial z$ (where subscripts denote averaging over the given direction); this is depicted in Fig. 5, which is produced from a 1-km-deep ABL simulation over an infinite sequence of cross-stream stripes of different surface roughness.

Figure 6 presents results from simulation CS1. Figure $6 a$ depicts the prescribed surface roughness. Figure $6 \mathrm{~b}$ is the normalized velocity, $u / u_{*}$, at the first plane of nodes above the surface at height $z=4.2 \mathrm{~m}$. The anticorrelation between the roughness and the velocity is very clear: rougher surfaces obviously reduce the speed of the flow. In Fig. 6b, we can also see the transition zones between the patches. Note that these zones are clearly visible in the streamwise direction, but they can be detected in the cross-stream direction as well. Also note that the lengths of such transition zones vary depending on the upstream and downstream roughness but these lengths are on the order of $100 \mathrm{~m}$ (note however the very long transition zone at about $x=y=2$ $\mathrm{km})$. Figure $6 \mathrm{c}$ depicts the wall stress. Very high stresses (about $-3 u_{*}^{2}$ ) are obtained at the upstream edges of rough patches and the stress is generally well correlated with surface roughness. The transition zones for the stress are shorter than for velocity, in agreement with Albertson and Parlange (1999a) and BMP04. This confirms that the IBLs based on stress propagate upward more rapidly than the ones based on velocity; therefore, stress data will give smaller transition regions at a given elevation but higher blending heights. The SGS dissipation is depicted in Fig. 6d. Like the stress, it is well correlated with the roughness: higher roughness produces more turbulence, which in turn leads to higher dissipation. One can see that the dissipation is very low above some smooth patches. This indicates that most of the turbulence is generated over the rough patches and confirms, along with the stress data, that these rough patches will be responsible for most of the momentum transfer at the earth surface-atmosphere interface.

Figure 7 is an instantaneous 3D slice plot of the normalized streamwise velocity for simulation CS1. The horizontal slice is at $4.2 \mathrm{~m}$ above ground and one can notice the signature of the varying patches even though no averaging is performed. Also notice the small variability scale at the surface confirming the small scale of the turbulent eddies in the vicinity of the ground. Finally, one can observe some "plumes" emanating from the surface and spreading downstream and carrying the signature of the different patches upward in the ABL.

\section{Parameterizations for effective surface roughness at a regional scale}

Figure 8 depicts the comparison of the blending height computed from the LES and predicted by the parameterization [Eq. (2)]; $L_{p}$ is computed from Eq. (6). The figure also shows the data from the simulations of BMP04 for comparison. The agreement of the results from the LES and the parameterization is very satisfactory and comparable to the agreement in BMP04. Note that the quality of the agreement does not seem to 

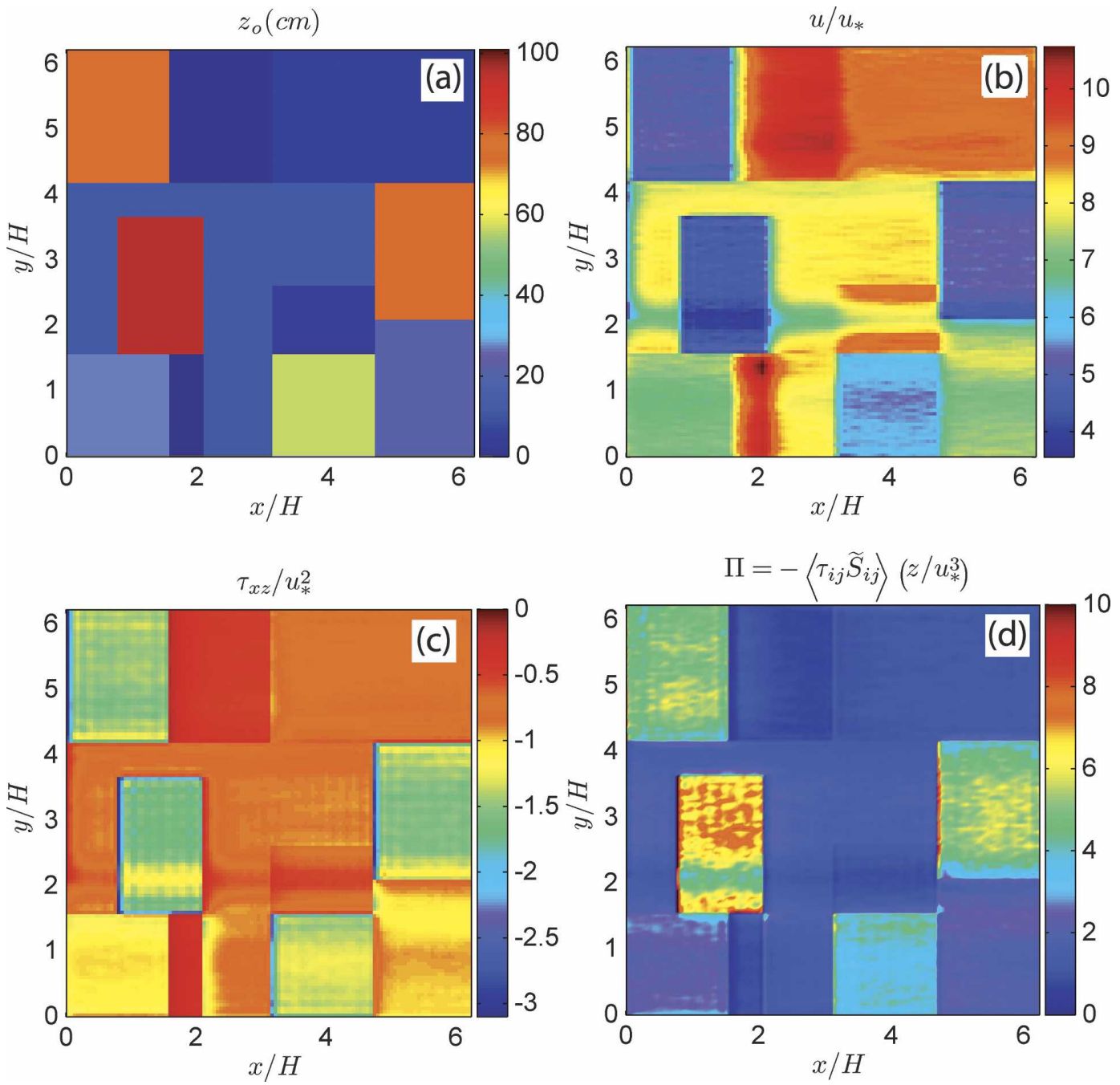

FIG. 6. (a) Surface roughness, (b) streamwise velocity at $z=4.2 \mathrm{~m}$, (c) wall stress, and (d) SGS dissipation at $z=4.2 \mathrm{~m}$ for the simulation CS1. Wind direction is from left to right.

depend on the blending height or the patch length. Figure 8 illustrates this good agreement between LES and model [Eq. (2)] results, as well as good collapse when normalizing $h_{b}$ and $L_{p}$ by $z_{o \text {,eff }}$. We also plotted the unnormalized predictions for $h_{b}$ versus those from the LES (not shown), and similarly found very good agreement. The correlation coefficient between the two (calculated over all 34 cases considered) is $R\left(h_{b, \text { Eq.(2) }}\right.$, $\left.h_{b, \mathrm{LES}}\right)=0.96$.

Figure 9a compares the effective surface roughness values obtained with the model and computed from LES. Since it is invariably the logarithm of the surface roughness that is used in similarity models and parameterizations, Fig. 9b shows the same comparison but for $\ln \left(z_{o, e}\right)$. As with the blending height, the agreement is good and the maximum error does not exceed $25 \%$ for any simu- lation. This error range is comparable to the simulations of BMP04, which are shown here for comparison.

Several other models have been proposed to compute an effective surface roughness for heterogeneous terrain by Taylor (1987, the log-average model), Mason (1988), Claussen (1990, 1991), and Goode and Belcher (1999). Here, we have tested the performance of all of these models in estimating the effective surface roughness computed from LES results. Note that, when testing the different models, we always used the characteristic heterogeneity scale of Eq. (6). This scale is required to apply the different parameterizations (except log-average) over realistic surfaces but no formulation for it has been proposed with any of the previous models. The different models have been invariably tested over simple configurations with one variability scale 


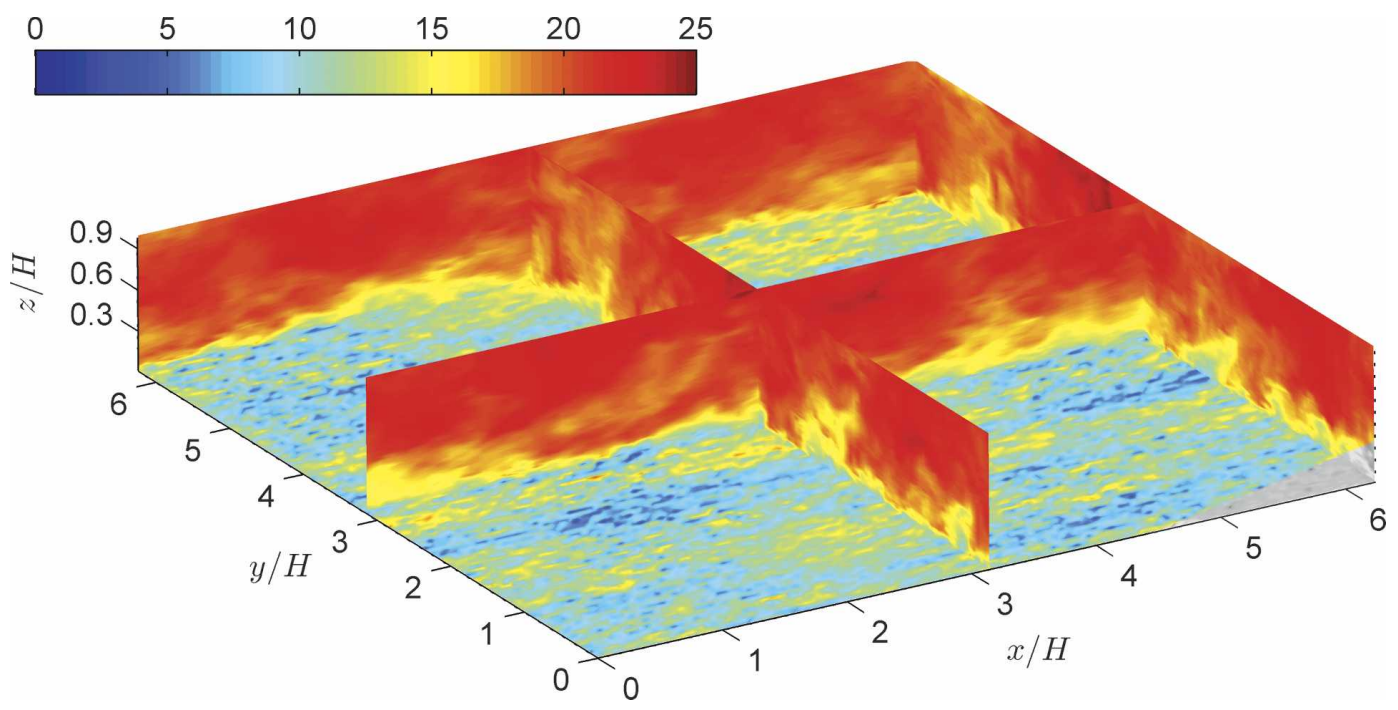

FIG. 7. Normalized streamwise velocity $\left(u / u_{*}\right)$ slices for simulation CS1. Horizontal slice at $z=4.2 \mathrm{~m}$.

consisting of the patch length. The use of the same variability scale with the different models partly explains the similar trends followed by the various models and confirms the suitability of the variability scale proposed here for the different parameterizations. As depicted in Fig. 10, the log-average model performs poorly, as illustrated also in BMP04. The shortcomings of the Mason model are not as obvious as in BMP04 mainly due to the smaller jumps in the roughness as compared to the tests in BMP04. The model proposed by Claussen $(1990,1991)$ unsurprisingly gives very similar results to BMP04 since, despite being derived using different approaches, the two parameterizations end up with quite similar equations for $z_{o, e}$ [the only difference is the -1 term in Eq. (3), which is only significant for short patches]. The Goode and Belcher parameterization gives similar overall performance in estimating $z_{o, e}$ compared to BMP04 and Claussen (1991). Nevertheless, the Goode and Belcher (1999) model is significantly more complex to implement and requires sim-

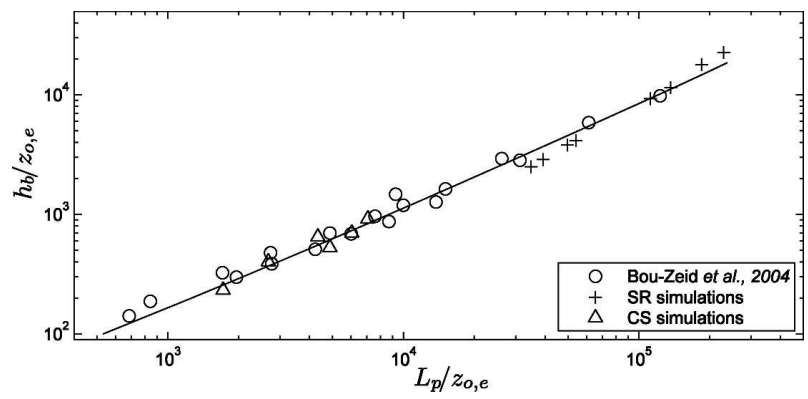

FIG. 8. Comparison of blending heights estimated by the analytical parameterization to LES values. Data points are LES results and the solid line is the prediction of the parameterization [Eq. (2)]. plifications in practice since it involves the estimation of the internal boundary layer (IBL) height at the end of each section of uniform roughness. We have been able to use this model for our highly complex patches only by making the additional simplification of using one IBL depth for all patches based on the integral variability scale [Eq. (6)].

These results answer the remaining questions: the BMP04 parameterization works well for complex patches and for surfaces with multiple variability scales when it is combined with the integral variability scale
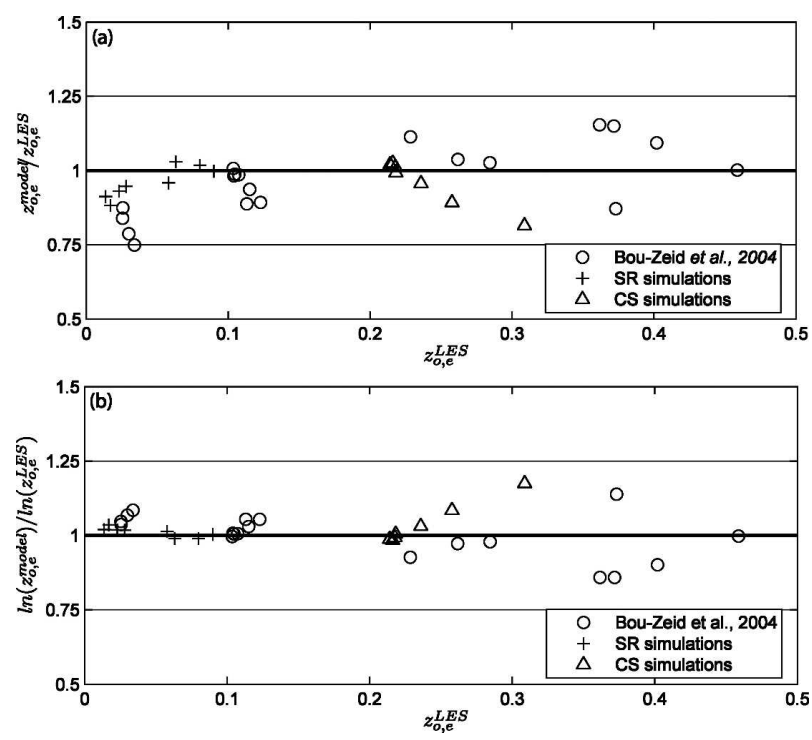

FIG. 9. Comparison of equivalent surface roughness estimated by the analytical parameterization in Eq. (3) to LES values. The thick line corresponds to $z_{o}^{\text {model }}=z_{o}^{\mathrm{LES}}$ (perfect fit): (a) error in $z_{o, e}$ and (b) error in $\ln \left(z_{o, e}\right)$. 


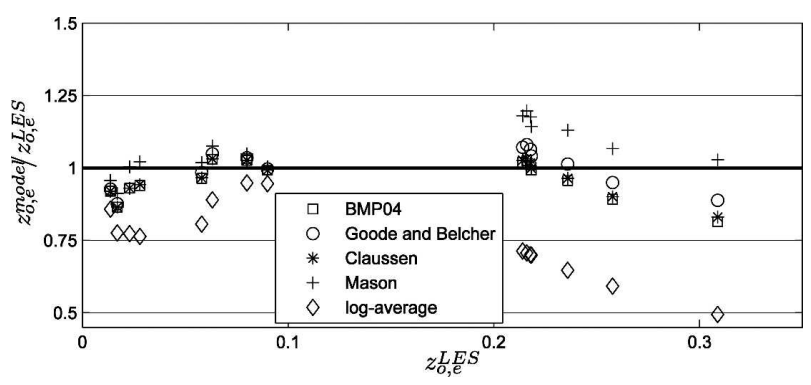

FIG. 10. Comparison of five different parameterizations for surface roughness to LES data.

proposed in Eq. (6). This variability scale is hence a good estimate of the average patch length needed in numerous regional-scale parameterizations.

\section{Conclusions}

This paper simulated ABL flow over complex distributions of rough patches. After an introduction discussing flow over variable surfaces and changes in roughness, a parameterization previously proposed by the authors (Bou-Zeid et al. 2004) was reviewed. The numerical code and the surfaces being simulated were then presented and the blending height and equivalent surface roughness were determined for all simulations. These simulations consisted of two sets: the first set had cross-stream stripes of unequal length and roughness. The second set consisted of surfaces with complex patch configurations; the surfaces were designed to have the same probability density function of surface roughness but with a decreasing variability scale. A measure of the spatial scale of surface variability was proposed based on an integral length scale formulation. Unlike definitions based on minima of correlation functions or maxima of variograms that pick the largest variability scale, the integral scale formulation gives an average value of the variability scale.

Basic results from the simulations depicted realistic variations of the velocity and stress close to the surface. Stresses were high over rough surfaces while velocities were high over smooth surfaces. The effect of variability in the cross-stream direction was found to be very limited. In addition, it was noted that the bulk of SGS dissipation occurred over the rough patches; dissipation over the smooth patches was insignificant. This suggested that most of the turbulence generation and momentum exchange occurred over the rougher patches.

The parameterization for the effective surface roughness and blending height proposed in Bou-Zeid et al. (2004) was combined with the characteristic variability scale formulation proposed in this paper and tested for the simulations of this study. The agreement was generally good and the error was about the same as for the simple patch configurations tested in Bou-Zeid et al. (2004). This confirms that the parameterization works well for complex patches and for surfaces with multiple variability scales and that the integral variability scale proposed here is a good estimate of the average patch length needed in the parameterization. The good performance also suggests that the basic premise of the parameterization, namely that blending is completed only when internal boundary layers have evolved over two times the characteristic horizontal variability scale, is sound.

Reduction in the variability scale was correlated with higher equivalent surface roughness values, indicating that variability enhances land-atmosphere interaction. This in turn confirms that any parameterization for effective surface roughness cannot rely only on singlepoint probabilistic distributions of surfaces properties but have to include a measure of variability (i.e., at least two-point statistics) to account for the effect of abrupt changes in surface roughness.

Finally, note that the parameterization proposed here assumes that the drag exercised by the individual patches can be accurately modeled using the law-ofthe-wall with a roughness $z_{o}$ for each patch. This entails that the patch size is significantly greater that the height of the roughness elements (buildings, trees ...) on the surface of the patch. A relevant issue not addressed in this paper is how to estimate the roughness length of these individual patches and how to account for flow adjustment through the individual roughness elements when the patch size is not significantly greater than the height of the roughness elements (see Belcher et al. 2003). Another useful extension of the current work would include the study of convective and stably stratified ABLs through the formulation of an effective Obukhov length scale, the effective surface roughness $z_{o, e}$ being, ideally, independent of stability.

Acknowledgments. This study was funded by NSF under EAR-9909679 and WCR-0233646 and by Agreement R828771-0-01 from the U.S. Environmental Protection Agency's Science to Achieve Results (STAR) program. The authors are thankful for the Scientific Computing Division of the National Center for Atmospheric Research (NCAR) for the use of their computer clusters.

\section{REFERENCES}

Albertson, J. D., and M. B. Parlange, 1999a: Natural integration of scalar fluxes from complex terrain. Adv. Water Resour., 23, 239-252.

_ , and _ 1999b: Surface length scales and shear stress: Implications for land-atmosphere interaction over complex terrain. Water Resour. Res., 35, 2121-2132.

Avissar, R., 1991: A statistical-dynamic approach to parameterize 
subgrid-scale land-surface heterogeneity in climate models. Surv. Geophys., 12, 155-178.

__ 1992: Conceptual aspects of a statistical-dynamic approach to represent landscape subgrid-scale heterogeneities in atmospheric models. J. Geophys. Res., 97, 2729-2742.

— , and R. A. Pielke, 1989: A parameterization of heterogeneous land surfaces for atmospheric numerical models and its impact on regional meteorology. Mon. Wea. Rev., 117, 2113-2136.

Belcher, S. E., N. Jerram, and J. C. R. Hunt, 2003: Adjustment of a turbulent boundary layer to a canopy of roughness elements. J. Fluid Mech., 488, 369-398.

Bou-Zeid, E., C. Meneveau, and M. B. Parlange, 2004: Largeeddy simulation of neutral atmospheric boundary layer flow over heterogeneous surfaces: Blending height and effective surface roughness. Water Resour. Res., 40, W02505, doi:10.1029/2003WR002475.

$\longrightarrow, \ldots$, and $—, 2005$ : A scale-dependent Lagrangian dynamic model for large-eddy simulation of complex turbulent flows. Phys. Fluids, 17, doi:10.1063/1.1839152.

Bradley, E. F., 1968: A micrometeorological study of velocity profiles and surface drag in region modified by a change in surface roughness. Quart. J. Roy. Meteor. Soc., 94, 361-379.

Brutsaert, W., 1998: Land-surface water vapor and sensible heat flux: Spatial variability, homogeneity, and measurement scales. Water Resour. Res., 34, 2433-2442.

Claussen, M., 1990: Area-averaging of surface fluxes in a neutrally stratified, horizontally inhomogeneous atmospheric boundary-layer. Atmos. Environ. A, 24, 1349-1360.

_ 1991: Estimation of areally-averaged surface fluxes. Bound.Layer Meteor., 54, 387-410.

Dai, Y., and Coauthors, 2003: The common land model. Bull. Amer. Meteor. Soc., 84, 1013-1023.

Deardorff, J. W., 1970: Preliminary results from numerical integrations of unstable planetary boundary layer. J. Atmos. Sci., 27, 1209-1211.

_ 1974: Three-dimensional numerical study of the height and mean structure of a heated planetary boundary layer. Bound.-Layer Meteor., 7, 81-106.

Germano, M., U. Piomelli, P. Moin, and W. H. Cabot, 1991: A dynamic subgrid-scale eddy viscosity model. Phys. Fluids A, 3, 1760-1765.

Goode, K., and S. E. Belcher, 1999: On the parameterisation of the effective roughness length for momentum transfer over heterogeneous terrain. Bound.-Layer Meteor., 93, 133-154.

Houghton, J. T., L. G. M. Filhom, D. J. Griggs, and K. Maskel, 1997: An introduction to simple climate models used in the IPCC second assessment report. International Panel on Climate Change, Working Group I, Tech. Paper, 39 pp.

Kleissl, J., C. Meneveau, and M. B. Parlange, 2003: On the magnitude and variability of subgrid-scale eddy-diffusion coefficients in the atmospheric surface layer. J. Atmos. Sci., 60, 2372-2388

—, M. B. Parlange, and C. Meneveau, 2004: Field experimental study of dynamic Smagorinsky models in the atmospheric surface layer. J. Atmos. Sci., 61, 2296-2307.

Koster, R. D., and M. J. Suarez, 1992: Modeling the land surface boundary in climate models as a composite of independent vegetation stands. J. Geophys. Res., 97, 2697-2715.

Kustas, W. P., and W. Brutsaert, 1986: Wind-profile constants in a neutral atmospheric boundary-layer over complex terrain. Bound.-Layer Meteor., 34, 35-54.

Lesieur, M., and O. Metais, 1996: New trends in large-eddy simulations of turbulence. Annu. Rev. Fluid Mech., 28, 45-82.
Lilly, D. K., 1967: The representation of small-scale turbulence in numerical simulation experiments. Proc. IBM Scientific Computing Symposium on Environmental Sciences, White Plains, NY, IBM, 195-209.

Lyons, T. J., and S. Halldin, 2004: Surface heterogeneity and the spatial variation of fluxes. Agric. For. Meteor., 121, 153-165.

Mason, P. J., 1988: The formation of areally-averaged roughness lengths. Quart. J. Roy. Meteor. Soc., 114, 399-420.

Meneveau, C., and J. Katz, 2000: Scale-invariance and turbulence models for large-eddy simulation. Annu. Rev. Fluid Mech., 32, 1-32.

_ T. S. Lund, and W. H. Cabot, 1996: A Lagrangian dynamic subgrid-scale model of turbulence. J. Fluid Mech., 319, 353-385.

Moeng, C. H., 1984: A large-eddy-simulation model for the study of planetary boundary-layer turbulence. J. Atmos. Sci., 41, 2052-2062.

Monin, A. S., and A. M. Obukhov, 1954: Basic laws of turbulent mixing in the ground layer of the atmosphere (in Russian). Tr. Geofiz. Inst., Akad. Nauk SSSR, 151, 163-187.

Orszag, S. A., 1970: Transform method for calculation of vectorcoupled sums-Application to spectral form of vorticity equation. J. Atmos. Sci., 27, 890-895.

_ 1971: On the elimination of aliasing in finite-difference schemes by filtering high-wavenumber components. J. Atmos. Sci., 28, 1074.

Parlange, M. B., and W. Brutsaert, 1989: Regional roughness of the Landes Forest and surface shear-stress under neutral conditions. Bound.-Layer Meteor., 48, 69-81.

_ , and — 1993: Regional shear-stress of broken forest from radiosonde wind profiles in the unstable surface-layer. Bound.-Layer Meteor., 64, 355-368.

_ W. E. Eichinger, and J. D. Albertson, 1995: Regional-scale evaporation and the atmospheric boundary-layer. Rev. Geophys., 33, 99-124.

Piomelli, U., 1999: Large-eddy simulation: Achievements and challenges. Prog. Aerosp. Sci., 35, 335-362.

Porte-Agel, F., C. Meneveau, and M. B. Parlange, 2000: A scaledependent dynamic model for large-eddy simulation: Application to a neutral atmospheric boundary layer. J. Fluid Mech., 415, 261-284.

Sagaut, P., 2006: Large Eddy Simulation for Incompressible Flows. $3 \mathrm{~d}$ ed. Springer-Verlag, $556 \mathrm{pp}$.

Shaw, R. H., and U. Schumann, 1992: Large-eddy simulation of turbulent-flow above and within a forest. Bound.-Layer Meteor., 61, 47-64.

Smagorinsky, J., 1963: General circulation experiments with the primitive equations. Part I: The basic experiment. Mon. Wea. Rev., 91, 99-164.

Stull, R. B., 1988: An Introduction to Boundary Layer Meteorology. Kluwer Academic Publishers, 670 pp.

Sugita, M., and W. Brutsaert, 1990: Wind velocity-measurements in the neutral boundary-layer above hilly prairie. J. Geophys. Res., 95, 7617-7624.

— soundings to obtain regional surface fluxes. Water Resour. Res., 28, 1675-1679.

Taylor, P. A., 1987: Comments and further analysis on effective roughness lengths for use in numerical 3-dimensional models. Bound.-Layer Meteor., 39, 403-418.

Wood, N., 2000: Wind flow over complex terrain: A historical perspective and the prospect for large-eddy modelling. Bound.-Layer Meteor., 96, 11-32. 